\title{
Tyrosinase Inhibition Kinetics of Anisic Acid
}

\author{
Isao Kubo ${ }^{a^{*},}$ Qing-Xi Chen ${ }^{a}$, Ken-Ichi Niheia , José S. Calderón ${ }^{b}$, and
}

Carlos L. Céspedes ${ }^{b^{*}}$

a Department of Environmental Science, Policy and Management, University of California, Berkeley, California 94720-3112, USA. Fax (510)643-0215.

E-mail: ikubo@uclink4.berkeley.edu

b Departamento de Productos Naturales, Instituto de Quimica, UNAM, Ciudad

Universitaria, Coyoacan, 04510 México, D. F., México. Fax: +52-55-5616-2203.

E-mail: ccespede@servidor.unam.mx

* Authors for correspondence and reprint requests

Z. Naturforsch. 58c, 713-718 (2003); received February 12/April 16, 2003

Anisic acid ( $p$-methoxybenzoic acid) was characterized as a tyrosinase inhibitor from aniseed, a common food spice. It inhibited the oxidation of L-3,4-dihydroxyphenylalanine (LDOPA) catalyzed by tyrosinase with an $\mathrm{IC}_{50}$ of $0.60 \mathrm{~mm}$. The inhibition of tyrosinase by anisic acid is a reversible reaction with residual enzyme activity. This phenolic acid was found to be a classical noncompetitive inhibitor and the inhibition constant $K_{\mathrm{I}}$ was obtained as $0.603 \mathrm{~mm}$. Anisic acid also inhibited the hydroxylation of L-tyrosine catalyzed by tyrosinase. The lag phase caused by the monophenolase activity was lengthened and the steady-state activity of the enzyme was decreased by anisic acid.

Key words: Tyrosinase Inhibitory Activity, Anisic Acid, Noncompetitive Inhibition Kinetics

\section{Introduction}

Tyrosinase (EC 1.14.18.1), also known as polyphenol oxidase (PPO) (Zawistowski et al., 1991; Whitaker, 1995; Mayer and Harel, 1998), is a copper containing mixed-function oxidase widely distributed in microorganisms, animals and plants. This oxidase catalyzes two distinct reactions of melanin synthesis, the hydroxylation of a monophenol and the conversion of an $o$-diphenol to the corresponding $o$-quinone (Robb, 1984). The hydroxylation of L-tyrosine, the initial step in melanin synthesis, is of considerable importance since it is also the initial step in catecholamine synthesis. Alterations in melanin synthesis occur in many disease states. Melanoma specific anticarcinogenic activity is also known being linked with tyrosinase activity (Prezioso et al., 1992). Melanins are also found in the mammalian eye and brain. Tyrosinase may play a role in neuromelanin formation in the human brain and could be central to dopamine neurotoxicity as well as contribute to the neurodegeneration associated with Parkinson's disease (Xu et al., 1997). Tyrosinase is also responsible for browning in plants and considered to be deleterious to the color quality of plant derived foods and beverages. This unfavorable darkening from enzymatic oxidation gen- erally results in a loss of nutritional and economic values and has been of great concern (Friedman, 1996). Similarly, the unfavorable browning caused by tyrosinase on the surface of seafood products has also been of great concern (Ogawa et al., 1984). In addition, tyrosinase inhibitors have become increasingly important in medicinal (Mosher et al., 1983) and cosmetic (Maeda and Fukuda, 1991) products in relation to hyperpigmentation. Hence, tyrosinase inhibitors should have broad applications.

By bioassay-guided fractionation using mushroom tyrosinase, anisaldehyde ( $p$-methoxybenzaldehyde) was characterized as the principal inhibitor from the $n$-hexane extract of the seeds of Pimpinella anisum L. (Umbelliferae) (Kubo and Kinst-Hori, 1998), commonly known as aniseed and widely used as a food spice. In our continuing search for tyrosinase inhibitors from plants (Kubo, 1997), the ethyl acetate fraction of the same plant was found to show the inhibitory activity and was subjected to further characterization.

\section{Material and Methods}

\section{General}

General procedures were the same as in the previous works (Kubo et al., 1994; Kubo and KinstHori, 1998). 


\section{Chemicals}

Anisic acid, p-ethoxybenzoate, $p$-propoxybenzoate, $p$-butoxybenzoate, 2-methoxybenzoic acid, 2,4-dimethoxybenzoic acid, and L-DOPA were purchased from Aldrich Chemical Co. (Milwaukee, WI). Benzoic acid, sodium dithionite, L-tyrosine, and dimethyl sulfoxide (DMSO) were obtained from Sigma Chemical Co. (St. Louis, MO).

\section{Enzyme assay}

The mushroom tyrosinase (EC 1.14.18.1) used for the bioassay was purchased from Sigma Chemical Co., and was used without further purification. Although mushroom tyrosinase differs somewhat from other sources (van Gelder et al., 1997), this fungal source was used for the entire experiment because it is readily available. The preliminarily assay was tested at $6.67 \mu \mathrm{g} / \mathrm{ml}$, unless otherwise specified. All the samples were first dissolved in DMSO and used for the experiment at 30 times dilution. The final concentration of DMSO in the test solution is $3.3 \%$. Tyrosinase functions both as a monophenolase and as an $o$-diphenolase. In the current experiment, L-DOPA was used as a substrate and hence, the activity studied is $o$-diphenolase inhibitory activity, unless otherwise noted. Tyrosinase catalyzes a reaction between two substrates, a phenolic compound and oxygen, but the assay was carried out in air-saturated solutions. The enzyme activity was monitored by dopachrome formation at $475 \mathrm{~nm}\left(\varepsilon=3700 \mathrm{M}^{-1} \mathrm{~cm}^{-1}\right)$ accompanying the oxidation of L-DOPA. One unit (U) of the enzymatic activity was defined as the amount of enzyme increasing 0.001 absorbance at $475 \mathrm{~nm}$ in the current experimental condition.

The diphenolase assay was performed as previously described (Kubo and Kinst-Hori, 1998) with slight modifications (Chen et al., 2000). First, $0.3 \mathrm{ml}$ of a $5.0 \mathrm{~mm}$ L-DOPA aqueous solution was mixed with $0.6 \mathrm{ml}$ of $0.25 \mathrm{M}$ phosphate buffer (pH 6.8) and $1.9 \mathrm{ml}$ water, incubated at $30{ }^{\circ} \mathrm{C}$ for $10 \mathrm{~min}$. Then, $0.1 \mathrm{ml}$ of the sample solution and $0.1 \mathrm{ml}$ of the aqueous solution of the mushroom tyrosinase $(0.2 \mathrm{mg} / \mathrm{ml}$ in $0.1 \mathrm{M}$ phosphate buffer, $\mathrm{pH}$ 6.8) were added in this order to the mixture. This solution was immediately monitored for the formation of dopachrome by measuring the linear increase in optical density at $475 \mathrm{~nm}$. The reaction was carried out under a constant temperature of
$30{ }^{\circ} \mathrm{C}$. Absorption measurements were recorded using a Spectra MAX plus Microplate spectrophotometer.

The monophenolase assay was performed with L-tyrosine as a substrate at $30{ }^{\circ} \mathrm{C}$. To the $3 \mathrm{ml}$ assay system containing $50 \mathrm{~mm}$ phosphate sodium buffer (pH 6.8), 2 mu L-tyrosine, different concentrations of anisic acid and $0.1 \mathrm{ml}$ of the enzyme $(1.0 \mathrm{mg} / \mathrm{ml}$ in $0.1 \mathrm{~m}$ phosphate buffer, $\mathrm{pH} 6.8$ ) was added, and the solution was immediately monitored by the optical density at $475 \mathrm{~nm}$, similar to the diphenolase assay.

\section{Measurement of oxygen consumption}

The reaction mixture consisted of $0.6 \mathrm{ml}$ of $0.25 \mathrm{M}$ phosphate buffer ( $\mathrm{pH} 6.8), 1.9 \mathrm{ml}$ of water and $0.1 \mathrm{ml}$ of $6 \mathrm{~mm}$ anisic acid or anisaldehyde DMSO solution was first incubated at $30^{\circ} \mathrm{C}$ for $5 \mathrm{~min}$. Then, $0.1 \mathrm{ml}$ of $0.05 \mathrm{M}$ phosphate buffer solution of mushroom tyrosinase $(20 \mu \mathrm{g})$ was added and oxygen consumption was measured with an $\mathrm{OBH} 100$ oxygen electrode and an oxygraph equipped with a water-jacket chamber of YSI 5300 (all from Yellow Springs Instruments Co., Yellow Springs, $\mathrm{OH}$ ) maintained at $30^{\circ} \mathrm{C}$ for $20 \mathrm{~min}$. A catalytic amount $(0.01 \mathrm{~mm})$ of L-DOPA was added after $5 \mathrm{~min}$. The oximeter was calibrated with air $(100 \%)$ and sodium dithionite $(0 \%)$.

\section{Results and Discussion}

The effect of anisic acid on the oxidation of L-DOPA catalyzed by mushroom tyrosinase was first studied. Anisic acid showed a dose-dependent inhibitory effect on this oxidation. As anisic acid increased, the enzyme activity was rapidly decreased but not completely suppressed. As the concentration of anisic acid reached to $2 \mathrm{~mm}$, the remaining enzymatic activity was about $24 \%$. The inhibitory concentration leading to $50 \%$ activity lost $\left(\mathrm{IC}_{50}\right)$ was estimated to be $0.60 \mathrm{~mm}$. This $\mathrm{IC}_{50}$ is nearly comparative to that of benzoic acid, a well-documented tyrosinase inhibitor (Conrad et al., 1994).

Subsequently, the inhibition mechanism of mushroom tyrosinase by anisic acid during the oxidation of L-DOPA was elucidated. The relationship of enzyme activity in the presence of different concentrations $(0,0.1,0.2,0.3$, and $0.4 \mathrm{~mm})$ of anisic acid. The results were a family of straight lines, 
which passed through the origin. Increasing the concentration of anisic acid resulted in the descending of the slopes of the lines, indicating that the inhibition of anisic acid on the enzyme was a reversible reaction course. The presence of anisic acid did not bring down the amount of the efficient enzyme, but resulted in the inhibition and the descending of the enzyme activity.

The inhibition kinetics of tyrosinase by anisic acid was investigated. The oxidation of L-DOPA catalyzed by tyrosinase follows Michaelis-Menten kinetics. The kinetic parameters for this enzyme obtained from a Lineweaver-Burk plot show that $K_{\mathrm{m}}$ is equal to $0.645 \mathrm{~mm}$ and $V_{\mathrm{m}}$ is equal to $138 \mathrm{~mm} / \mathrm{min}$. It should be noted however that tyrosinase catalyzes a reaction between two substrates, a phenolic compound and oxygen, but the assay was carried out in air-saturated aqueous solutions. Therefore, $K_{\mathrm{m}}$ and $V_{\mathrm{m}}$ values determined in this condition were only apparent, and the effect of oxygen concentration on these parameters is unknown.

The kinetic behavior of the oxidation of L-DOPA by mushroom tyrosinase at different concentrations of anisic acid, indicates that anisic acid is a classical noncompetitive inhibitor to the enzyme (Fig. 1). It can decrease the apparent value of $V_{\mathrm{m}}$ with no effect on that of $K_{\mathrm{m}}$. This behavior is observed that anisic acid can combine with both free

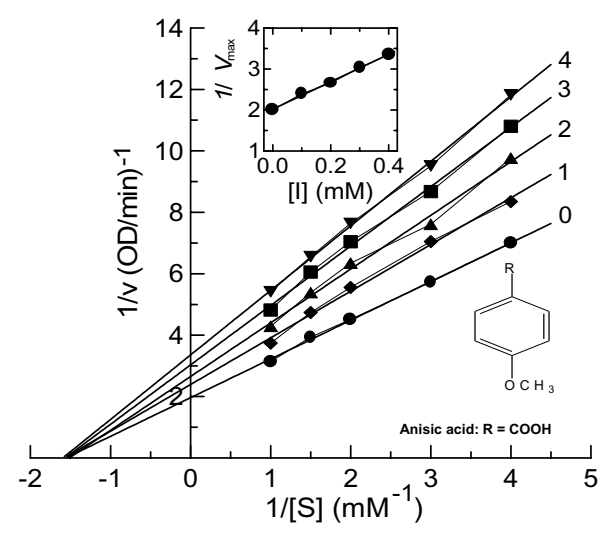

Fig. 1. Lineweaver-Burk plots for inhibition of anisic acid on mushroom tyrosinase for the catalysis of L-DOPA at $30{ }^{\circ} \mathrm{C}$. Concentration of anisic acid for curves $0-4$ was $0,0.1,0.2,0.3$, and $0.4 \mathrm{~mm}$, respectively. The inset represents the plot of $1 / V_{\max }$ versus the anisic acid concentration for determining the inhibition constants $K_{\mathrm{I}}$. The line was drawn using linear least squares fit. Chemical structure of anisic acid and its related phenolics. enzyme and the enzyme-substrate complex, and there are same binding intensity between anisic acid and both of the enzyme forms.

The equilibrium constants for inhibitor binding with free enzyme $(\mathrm{E}), K_{\mathrm{I}}$, and with enzyme-substrate (ES) complex, $K_{\mathrm{IS}}$, are the same in quantity, which can be obtained from the second plot of the "Intercept" versus concentration of inhibitor, which are linears (Fig. 1).

In addition, $p$-ethoxybenzoic acid, p-propoxybenzoic acid, and $p$-butoxybenzoic acid were also tested for their mushroom tyrosinase inhibitory activity emphasizing their inhibition type for comparison. As a result, the oxidation of L-DOPA catalyzed by mushroom tyrosinase was inhibited by these $p$-alkoxybenzoates and the inhibitory activity decreased with increasing carbon chain length. Interestingly, the inhibition type also changed with increasing carbon chain length as noncompetitive, mixed type, and, presumably to competitive inhibitors. Neither 2-methoxybenzoic acid and nor 2,4dimethoxybenzoic acid exhibited any inhibitory activity up to $5 \mathrm{~mm}$.

Tyrosinase contains functions both as a monophenolase and an $o$-diphenolase. The discussion so far described is based on the experiment using L-DOPA as a substrate. Therefore, the activity mentioned is $o$-diphenolase inhibitory activity of mushroom tyrosinase. On the other hand, the lag time is known for the oxidation of monophenol substrates such as L-tyrosine to L-DOPA. This lag time can be extended by monophenolase inhibitors such as tropolone (Kahn and Andrawis, 1985) and galangin (Kubo and Kinst-Hori, 1998). Anisic acid was noted to inhibit the monophenolase of mushroom tyrosinase. The time course of the oxidation of L-tyrosine (substrate) in the presence of different anisic acid concentrations is shown in Fig. 2. At each concentration of anisic acid, the lag period of L-tyrosine oxidation by tyrosinase was lengthened with increasing the concentration of this inhibitor. The steady-state rate was also influenced. As the concentration of anisic acid increased, the steady-state activity of the enzyme was rapidly decreased. As the concentration of anisic acid reached to $4 \mathrm{~mm}$, the remaining enzymatic activity was about $20 \%$. The inhibitory concentration leading to $50 \%$ activity lost $\left(\mathrm{IC}_{50}\right)$ was estimated to be $2.20 \mathrm{~mm}$. 


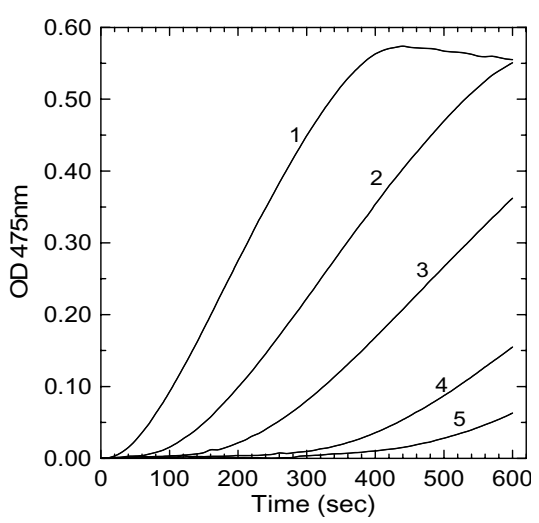

Fig. 2. Effects of anisic acid on the activity of mushroom tyrosinase for the oxidation of L-tyrosine at $30^{\circ} \mathrm{C}$. The reaction media $(3 \mathrm{ml})$ contained $0.5 \mathrm{~mm}$ of tyrosine in 50 mu sodium phosphate buffer ( $\mathrm{pH} 6.8$ ), mushroom tyrosinase $33.3 \mu \mathrm{g} / \mathrm{ml}$. The concentration of anisic acid for curves $1-6$ was $0,0.4,1.0,2.0,3.0$ and $4.0 \mathrm{~mm}$, respectively.

In the different concentration of L-tyrosine, the lag period of the oxidation reaction was lengthened by anisic acid in different degree, as shown in Fig. 3. When the concentration of tyrosine was low, the lag period was lengthened by anisic acid by a greater degree. Increasing the concentration of monophenol resulted that the degree of lengthening lag period became weaker, indicating that the substrate offers marked protection of the enzyme against inhibition by anisic acid. It also can be seen from reducing the decreasing the steadystate activity of the enzyme in the presence of anisic acid.

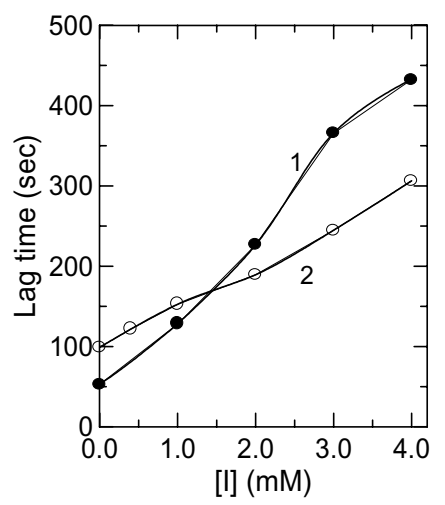

Fig. 3. Effect of monophenol concentration on lengthening the lag time of mushroom tyrosinase by anisic acid. The concentrations of L-tyrosine for curves 1 and 2 were 0.5 and $2.0 \mathrm{~mm}$, respectively.
Table I. Kinetic and inhibition constants of anisic acid and anisaldehyde.

\begin{tabular}{lll}
\hline Constants & Anisic acid & Anisaldehyde \\
\hline $\mathrm{IC}_{50}$ & $0.60 \mathrm{~mm}$ & $0.40 \mathrm{~mm}$ \\
$K \mathrm{M}$ & $0.645 \mathrm{~mm}$ & $0.664 \mathrm{~mm}$ \\
$V_{\mathrm{m}}$ & $0.138 \mathrm{~mm} / \mathrm{min}$ & $0.125 \mathrm{~mm} / \mathrm{min}$ \\
Inhibition & reversible & reversible \\
Inhibition type & noncompetitive & noncompetitive \\
$K_{\mathrm{I}}$ & $0.603 \mathrm{~mm}$ & $0.376 \mathrm{~mm}$ \\
\hline
\end{tabular}

Anisaldehyde isolated from the same source was previously reported as a noncompetitive inhibitor (Kubo and Kinst-Hori, 1998), its inhibition mode was also reinvestigated. The result obtained by the current kinetic study confirmed that the inhibition mode of anisaldehyde is the same as before. The kinetic constants obtained are summarized in Table I. In addition, anisaldehyde did not inhibit the hydroxylation of L-tyrosine (monophenolase activity) the same as previously reported (Kubo and Kinst-Hori, 1998). This result is different from that of anisic acid being described above. In brief, anisic acid inhibits monophenolase activity but anisaldehyde does not.

As above mentioned, tyrosinase is known to catalyze a reaction between two substrates, a phenolic compound and oxygen. The discussion so far described is based on the experiment using mainly L-DOPA as a substrate. As the need arises, L-tyrosine was also assayed to see if the inhibitors characterized were effective on monophenolase activity. Consequently, the enzyme activity was monitored by dopachrome formation at $475 \mathrm{~nm}$. On the other hand, the fact that oxygen is the other substrate needs to be considered. Hence, the enzyme activity was also monitored by oxygen consumption to examine it from a different angle. The mixture consisted of tyrosinase $(20 \mu \mathrm{g})$ and $0.2 \mathrm{~mm}$ of the samples was incubated and oxygen consumption was measured for $20 \mathrm{~min}$. The arrow indicates the time when catalytic amount (0.01 mu) of L-DOPA was added. The results obtained are shown in Fig. 3. As expected, neither anisic acid nor anisaldehyde consumed oxygen at all even after adding L-DOPA. The results are consistent with the data observed by monitoring dopachrome formation.

The current kinetic study of tyrosinase inhibition found that anisic acid is a noncompetitive in- 
hibitor and leads to reversible inhibition with residual enzyme activity. The results obtained are consistent with those of the previous report (Kubo and Kinst-Hori, 1998). The kinetic behaviors of the $p$-alkoxybenzoic acids tested are similar to anisic acid.

According to previous papers (Conrad et al., 1994), as long as a free carboxyl group exists in the aromatic ring, hydroxyphenolic acids such as para- and meta-hydroxybenzoic acid bind to the binuclear copper active center presumably with the more acidic carboxylic group. These hydroxyphenolic acids are coordinated to one copper as if monophenol substrates and are positioned over the binuclear active site, and inhibit the enzyme competitively (Wilcox et al., 1985). However, this may not be the case for anisic acid. Although the precise explanation how these benzoic acid derivatives interact with the enzyme on a molecular basis is still largely unknown, anisic acid may disrupt the tertiary structure of the enzyme through intermolecular hydrogen bonding. This is more likely as a non-competitive inhibitor, in part, reducing the

Chen Q. X., Zhang Z., Zhou X. W., and Zhuang Z. L. (2000), Kinetics of inhibition of $\beta$-glucosidase from Ampullarium crossean by bromoacetic acid. Int. J. Biochem. Cell Biol. 32, 717-723.

Conrad J. S., Dawso S. R., Hubbard E. R., Meyers T. E., and Strothkamp K. G. (1994), Inhibitor binding to the binuclear active site of tyrosinase: Temperature, $\mathrm{pH}$, and solvent deuterium isotope effects. Biochemistry 33, 5739-5744.

Friedman M. (1996), Food browning and its prevention: An overview. J. Agric. Food Chem. 44, 631-653.

Kahn V. and Andrawis A. (1985), Inhibition of mushroom tyrosinase by tropolone. Phytochemistry 24, 905-908.

Kubo I. (1993), Insect control agents from tropical plants. In: Recent Advances in Phytochemistry, Phytochemical Potential of Tropical Plants (K. R. Downum, J. T. Romeo, and H. A. Stafford, eds.). Plenum, New York, Vol. 27, pp. 133-151.

Kubo I. (1997), Tyrosinase inhibitors from plants. In: Phytochemicals for Pest Control (P. Hedin, R. Hollingworth, E. Masler, J. Miyamoto, and D. Thompson, eds.). ACS Symposium Series 658, American Chemical Society, Washington, DC, pp. 311-326.

Kubo I. and Himejima M. (1991), Anethole, a synergist of polygodial against filamentous microorganisms. J. Agric. Food Chem. 39, 2290-2292. affinity of the substrates with the enzyme (Jackman et al., 1992). This indicates that the carboxylic group is involved in the hydrogen-bonding interactions. Thus, anisic acid may disrupt this interaction.

The observation that neither 2-methoxybenzoic acid nor 2,4-dimethoxybenzoic acid exhibited any inhibitory activity supports the postulate because of the steric hindrance. The low conformational stabilities of native proteins make them easily susceptible to denaturation by altering the balance of the weak nonbonding forces that maintain the native conformation.

\section{Acknowledgements}

This material is based upon the work supported in part by a grant from the University of California Institute for Mexico and the United States (UC MEXUS) and the Consejo Nacional de Ciencia y Tecnología de México (CONACYT). Q. X. Chen thanks the Lee Foundation and the Berkeley Scholars Program for financial support during his study at the University of California, Berkeley.
Kubo I., Kinst-Hori I., and Yokokawa Y. (1994), Tyrosinase inhibitors from Anacardium occidentale fruits. J. Nat. Prod. 57, 545-551.

Kubo I. and Kinst-Hori I. (1998), Tyrosinase inhibitors from anise oil. J. Agric. Food Chem. 46, 1268-1271.

Maeda K. and Fukuda M. (1991), In vitro effectiveness of several whitening cosmetic components in human melanocytes. J. Soc. Cosmet. Chem. 42, 361-368.

Mayer A. M. and Harel E. (1998), Phenoloxidases and their significance in fruit and vegetables. In: Food Enzymology (P. F. Fox, ed.). Elsevier, London, UK, pp. 373-398.

Mosher D. B., Pathak M. A., and Fitzpatrick T. B. (1983), Vitiligo, etiology, pathogenesis, diagnosis, and treatment. In: Update: Dermatology in General Medicine (T. B. Fitzpatrick, A. Z. Eisen, K. Wolff, I. M. Freedberg, and K. F. Austen, eds.). McGraw-Hill, New York, pp. 205-225.

Ogawa M., Perdigao N. B., Santiago M. E., and Kozima T. T. (1984), On physiological aspects of black spot appearance in shrimp. Bull. Jpn. Soc. Sci. Fish. 50, $1763-1769$.

Prezioso J. A., Epperly M. W., Wang N., and Bloomer W. D. (1992), Effects of tyrosinase activity on the cytotoxicity of $4-S$-cysteaminylphenol and $N$-acetyl-4-Scysteaminylphenol in melanoma cells. Cancer Lett. 63, $73-79$. 
Robb D. A. (1984), Tyrosinase. In: Copper Proteins and Copper Enzymes (R. Lontie, ed.). CRC Press, Florida, Vol. II, pp. 207-240.

Van Gelder C. W. G., Flurkey W. H., and Wichers, H. J. (1997), Sequence and structural features of plant and fungal tyrosinases. Phytochemistry 45, 1309-1323.

Whitaker J. R. (1995), Polyphenol oxidase. In: Food Enzymes, Structure and Mechanism (D. W. S. Wong, ed.). Chapman \& Hall, New York, pp. 271-307.

Wilcox D. E., Porras A. G., Hwang Y. T., Lerch K., Winkler M. E., and Solomon E. I. (1985), Substrate analogue binding to the coupled binuclear copper active site in tyrosinase. J. Am. Chem. Soc. 107, $4015-4027$.

Xu Y., Stokes A. H., Freeman W. M., Kumer S. C., Vogt B. A., and Vrana K. E. (1997), Tyrosinase mRNA is expressed in human substantia nigra. Mol. Brain Res. 45, 159-162.

Zawistowski J., Biliaderis C. G., and Eskin N. A. M. (1991), Polyphenol oxidase. In: Oxidative Enzymes in Foods (D. S. Robinson and N. A. M. Eskin, eds.). Elsevier, London, pp. 217-273. 